\title{
Lithographical Fabrication of Organic Single-Crystal Arrays by Area-Selective Growth and Solvent Vapor Annealing
}

Hong Wang ${ }^{1,3}$, Florian Fontein ${ }^{1}$, Jianping $\mathrm{Li}^{2}$, Lizhen Huang ${ }^{2}$, Lin Jiang ${ }^{2}$, Harald Fuchs $^{1}$, Wenchong Wang ${ }^{1}$, Yandong Wang ${ }^{2}$ and Lifeng $\mathrm{Chi}^{2} *$

1. Physikalisches Institut and Center for Nanotechnology (CeNTech), Universität Münster, 48149 Münster, Germany.

2. Jiangsu Key Laboratory for Carbon-Based Functional Materials \& Devices, Institute of Functional Nano \& Soft Materials (FUNSOM), Joint International Research Laboratory of Carbon-Based Functional Materials and Devices, Soochow University, 199 Ren'ai Road, Suzhou, 215123, Jiangsu, P.R. China.

3. School of Materials and State Key Laboratory of Optoelectronic Materials and Technologies, Sun Yat-sen University, 135 Xingang Xi Road, Guangzhou, 510275, Guangdong, P. R. China.

*Corresponding author. E-mail: wangw@uni-muenster.de;

wangyandong@suda.edu.cn; chilf@suda.edu.cn 


\section{Figures:}
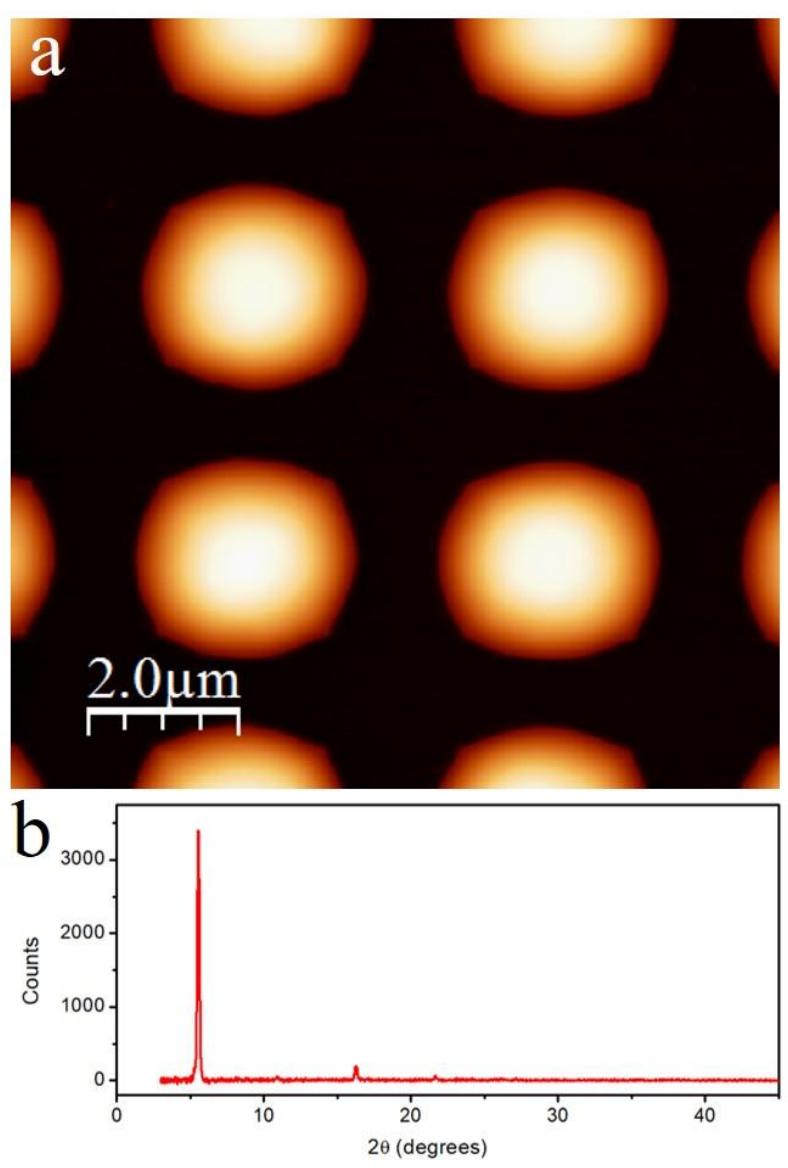

Figure S1 (a) AFM image and (b) XRD patterns of the sample after deposition of 12 nm TES-ADT on Au patterned $\mathrm{SiO}_{2}$ surface where the substrate temperature was kept at $155^{\circ} \mathrm{C}$. 

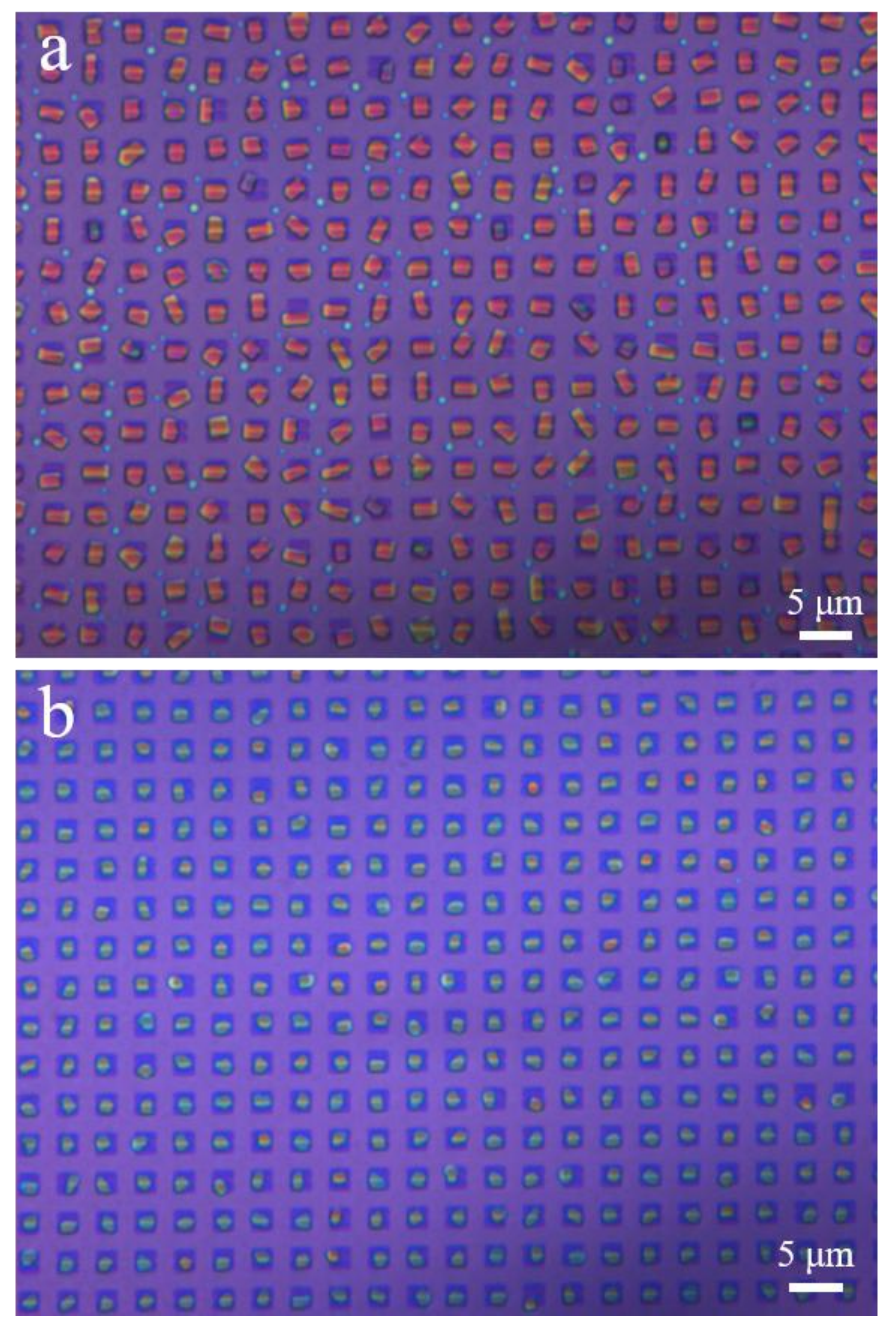

Figure S2 Optical microscope images of (a) $12 \mathrm{~nm}$ and (b) 6nm TES-ADT deposited on Au array with periodicity of $2 \mu \mathrm{m}$ after SVA. Obviously, the size of the crystals obtained by depositing $6 \mathrm{~nm}$ TES-ADT is smaller than that of $12 \mathrm{~nm}$ TES-ADT. 


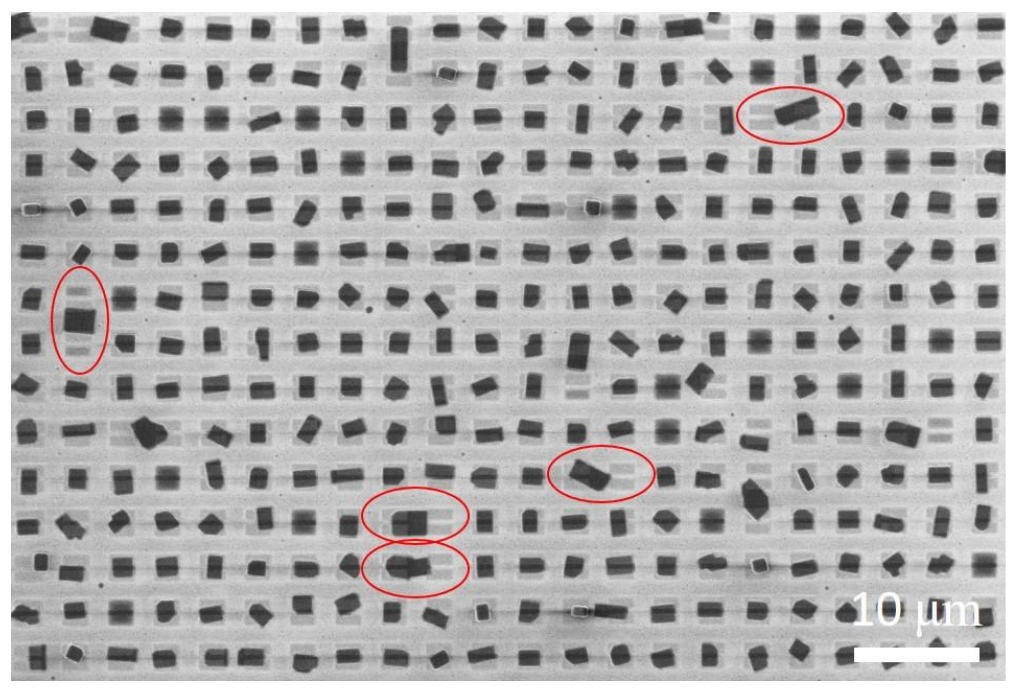

Figure S3 SEM image of the sample after deposition of $12 \mathrm{~nm}$ TES-ADT and subsequent SVA. Most of the electrode pairs are covered by TES-ADT single crsytals. Some electrode pairs covered by big crystal due to merging of neibourghing droplets, leaving electrode pairs not occupied by crystal as marked by red ellipses. 

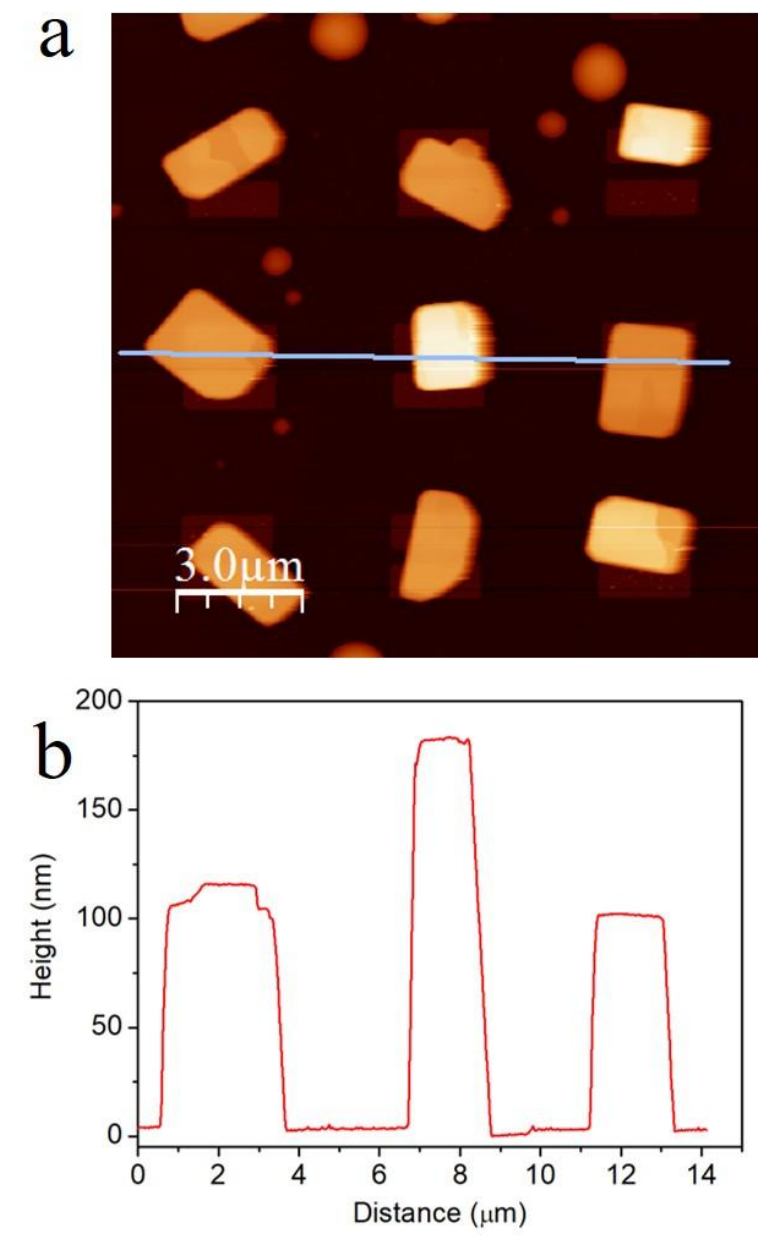

Figure S4 (a) AFM image of TES-ADT crystals on Au electrode pair array with the spacing of $2.1 \mu \mathrm{m}$. (b) Height profile of the line marked in (a), where the heights of the three crystals are $115.7 \mathrm{~nm}, 182.4 \mathrm{~nm}$ and $102.3 \mathrm{~nm}$, respectively. The hight of the middle one is much larger than the other two, where the corresponding crystal shows green color in optical microsocope image. 

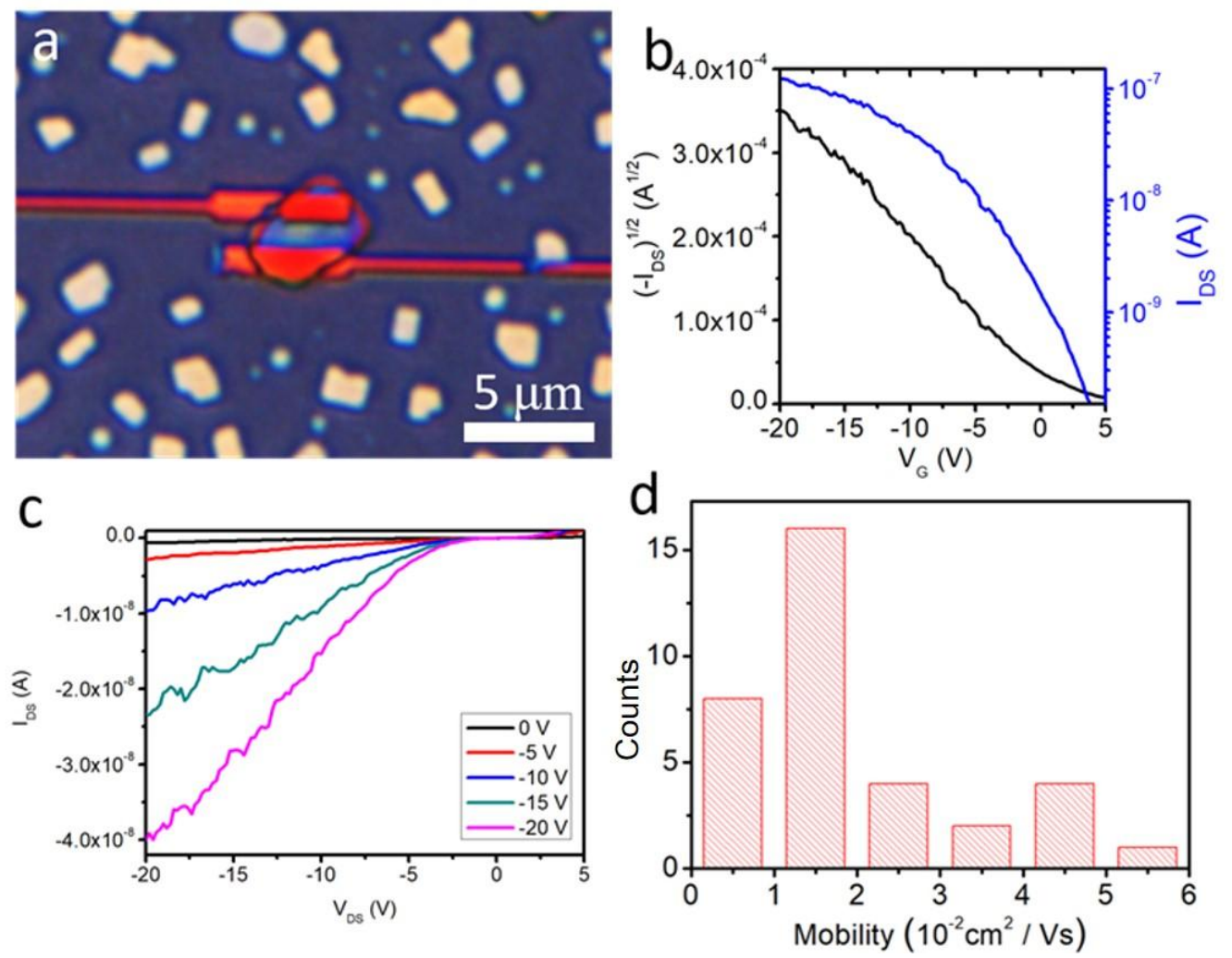

Figure S5 (a) Optical microscope image of a fabricated OFET device based on a TES-ADT single crystal. (b) Transfer curves and (c) output curves of a typical OFET device. (d) Histogram of hole carrier mobility of OFET devices based on TES-ADT single crsytals. The length and width of the channel are $1 \mu \mathrm{m}$ and $5 \mu \mathrm{m}$ respectively. 

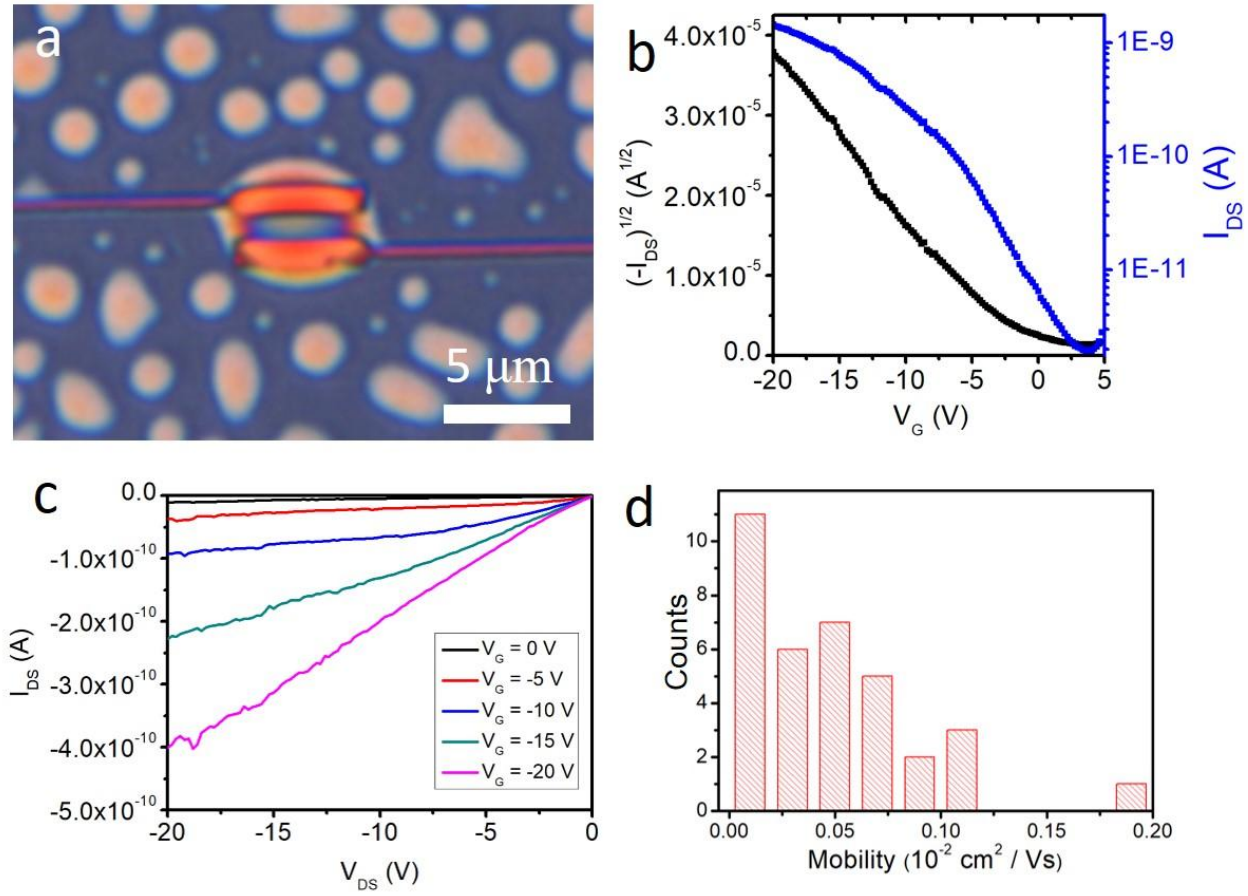

Figure S6 (a) Optical microscope image of an as grown TES-ADT OFET. (b) Transfer curves and (c) output curves of a typical OFET device. (d) Histogram of hole carrier mobility of OFET devices based on as grown droplets. 